\title{
DINASTÍNEOS (COLEOPTERA, SCARABAEOIDEA, MELOLONTHIDAE) EM UMA ÁREA DE TERRA FIRME NA AMAZÔNIA CENTRAL, BRASIL ${ }^{1}$
}

\author{
Ricardo ANDREAZZE ${ }^{2}$, Claudio Ruy V. FONSECA ${ }^{3}$
}

RESUMO - Foram efetuadas coletas mensais de julho/1990 a junho/1991, durante a lua nova, na Fazenda Aruanã, uma área de terra-firme às margens da rodovia Torquato Tapajós, AM-010, $\mathrm{Km}$ 215, Município de Itacoatiara, Estado do Amazonas, Brasil. Utilizou-se para as coletas luz mista de mercúrio de $250 \mathrm{~W}$, sobre um lençol branco. Foram coletados 251 indivíduos de 9 gêneros e 18 espécies de dinastíneos e dentre estes houve predominância de Cyclocephala Latreille (3 espécies abundantes) e Ligyrus Burmeister (única espécie abundante).

Palavras chaves: Coleoptera, Scarabaeoidea, Melolonthidae, Dynastinae, Amazônia Central.

Dynastinae Beetle (Coleoptera, Scarabaeoidea, Melolonthidae) of an Upland Rainforest Area in Central Amazonia, Brazil.

ABSTRACT - Monthly collections were done between July/1990 and June/1991 during the new moon at the Fazenda Aruanã an area of upland rainforest located alongside the Torquato Tapajós road, AM-010, at Km 215, city of Itacoatiara, Amazonas State, Brazil. A mixed $250 \mathrm{~W}$ mercury vapor lamp was used for atraction on a white sheet. 251 specimens of 9 genera and 18 species of Dynastinae were collected and there was predominance of Cyclocephala (three species were more numerous) and Ligyrus (only one abundant).

Key words: Coleoptera, Scarabaeoidea, Melolonthidae, Dynastinae, Amazon Basin.

\section{INTRODUÇÃO}

Os besouros da superfamília Scarabaeoidea caracterizam-se por possuírem antenas lameladas (Lima, 1953; Ritcher, 1958). A organização dos Dynastinae tem sido alvo de várias modificações nos últimos anos e as classificações propostas são várias. No presente trabalho adotamos Dynastinae como uma subfamília de Melolonthidae segundo o critério de Endördi (1966 apud Morón, 1981) que considera Lamellicornia subdividida em 5 famílias: Lucanidae, Passalidae, Trogidae (Scarabaeidae - Troginae de
Janssens, 1946), Scarabaeidae (Scarabaeidae - Laparosticti de Janssens, 1946) e Melolonthidae (Scarabaeidae Pleurosticti de Janssens, 1949).

Os dinastíneos são um grupo importante dentre os coleópteros de atividade decompositora, principalmente os considerados como a macrofauna da floresta (tamanho $>1 \mathrm{~cm}$ ) porque durante a sua alimentação eles fragmentam os restos vegetais ou animais, produzindo detritos e excretas que expõem uma maior superfície para a ação de outros decompositores, representados pela microfauna e microflora (Morón, 1985).

I Trabalho apresentado à Coordenação do Programa de Pós-Graduação em Biologia Tropical e Recursos Naturais do Convênio INPA/FUA como parte dos requisitos para obtenção do título de Mestre em Ciências Biológicas, Área de Concentração em Entomologia.

2 Estudante de doutorado no Programa de Pos-Graduação INPA/FUA. C.P. $47860911-970$ Manaus AM

3 Pesquisador Titular do INPA/CPEN. C.P. 478 69011-970 Manaus AM 
Entre os Dynastinae, alguns dos gêneros se destacam pela importância agrícola. Cyclocephala possui mais de 200 espécies com distribuição nas Américas, principalmente na região tropical (Blackwelder, 1944; Endrödi, 1985), sendo algumas espécies consideradas pragas agrícolas de culturas como milho, cana-de-açúcar e frutas como maracujá ou goiaba (Gallo et al., 1978; Morón 1984; Morón et al., 1985). Ligyrus possui 26 espécies conhecidas, distribuídas por todas Américas (Blackwelder, 1944; Endrödi,1985). Algumas de suas espécies são conhecidas por provocarem danos a certas hortaliças, milho, sorgo (Morón et al., 1985) e principalmente a cana-deaçúcar, sendo as larvas conhecidas como "pão-de-galinha" (Lima, 1953; Gallo et al., 1978; Morón et al., 1985).

A atração dos insetos à luz ainda é um fenômeno discutido e tem sido objeto de curiosidade e estudo há bastante tempo, assim como o efeito da luz lunar em coletas noturnas (Williams, 1936; Williams \& Singh, 1951).

Coletas com lençol suspenso e lâmpada de luz mista de mercúrio para os Dynastinae ainda não têm registros em áreas na Amazônia. Encontramos dados quanto a coletas de Passalídeos por esse mesmo método de captura no rio Uraricoera, Ilha de Maracá, Roraima (Bührnheim \& Aguiar, 1991), e também no alto rio Urubu, município de Presidente Figueiredo, Amazonas (Aguiar \& Bührnheim, 1992). No México, utilizaram a lâmpada sobre tecido branco porém com luz fluorescente vertical para a captura de dinastíneos (Morón, 1984).
O objetivo deste trabalho foi investigar a fauna de dinastíneos que ocorreu durante um ano numa área de terra firme, coletada com lâmpada de luz mista de mercúrio em períodos de lua nova e observar as diferentes espécies que ocorreram, identificá-las e compará-las. Quanto ao método de coletas, avaliar sua eficiência em relação a esta família, a partir de registros quantitativos e qualitativos das espécies no ano.

\section{MATERIAL E MÉTODOS}

O estudo foi realizado na Fazenda Aruanã, situada às margens da rodovia Torquato Tapajós, AM-010, Km 215, Municipio de Itacoatiara, tendo como coordenadas geográficas aproximadas $3^{\circ} 02^{\prime} \mathrm{S}$ e $58^{\circ} 50^{\prime} \mathrm{W}$. Os limites da fazenda estão em terra-firme em uma extensa área (17 km X $25 \mathrm{~km})$, sendo grande parte da área, cerca de $70 \%$, ainda ocupada por floresta primária, com terreno acidentado, irrigada com grande número de igarapés. O projeto agropecuário inicial da Fazenda Aruanã, há cerca de 20 anos, era uma tentativa de implantação de pastagens a partir da derrubada e queima da mata (Dantas, 1979).

Nos últimos 10 anos a atividade econômica principal da fazenda apresentou mudanças reduzindo-se os rebanhos e aproveitando-se as extensas áreas derrubadas para o plantio de algumas variedades de castanheira (Bertholletia excelsa Humb. \& Bonpl.).

A sede da fazenda foi escolhida como local para as coletas onde armou-se o lençol e a lâmpada em uma das paredes externas com face 
voltada para a floresta a uma distância aproximada de 200 metros.

Foi utilizado um lençol branco de $1,40 \times 2,20 \mathrm{~m}$, com uma lâmpada de luz mista de mercúrio de $220 \mathrm{~V}, 250$ $\mathrm{W}$, alimentada por um motor gerador de $1500 \mathrm{~W}$. O lençol foi aberto na vertical, encostado à parede com sua extensão maior na horizontal e suspenso a $2 \mathrm{~m}$ do chão com a lâmpada acima deste em um soquete com suporte protetor. Na borda inferior do lençol, fez-se uma dobra de 15 $\mathrm{cm}$ aproximadamente, formando uma canaleta onde se acumulavam os insetos. Este método é uma modificação do inicialmente utilizado por Laroca \& Mielke (1975) para coletas de lepidópteros.

As coletas extenderam-se por 3 noites consecutivas em intervalos de 12 horas seguidas (das 18:00 às 06:00 h) antes e durante o período de lua nova, periodo esse em que há menos interferência da luz lunar (Williams, 1936; Williams \& Singh, 1951).

As atividades iniciaram-se em julho de 1990 e cessaram em junho de 1991, coletando-se durante 3 noites consecutivas nos períodos de lua nova.

Os dinastíneos foram capturados manualmente, um a um, assim que pousavam no lençol e colocados em um frasco mata-inseto contendo algodão embebido com acetato de etila, coberto por papel absorvente. Os exemplares maiores foram retirados do frasco e envoltos com papel absorvente e acondicionados em sacos plásticos com rótulos provisórios. No laboratório, os insetos foram montados em alfinetes entomológicos, dessecados em estufa a $50^{\circ} \mathrm{C}$ durante 48 horas, etiquetados e identificados. A identificação do material, machos e fềmeas, foi feita utilizando-se chaves de identificação para espécies de Dynastinae, de acordo com Endrödi (1985). Para os machos, utilizou-se também a genitália na identificação.

O dimorfismo sexual entre dinastíneos pode ser observado por alguns caracteres, por ex., garras tarsais anteriores diferenciadas $\left(\sigma^{\circ}\right)$, chifres ou pelo último segmento abdominal, o pigídio, o qual apresenta nos machos uma pequena incisão em forma de meia lua na sua porção mediana.

A partir da separação dos sexos entre os indivíduos procedeu-se a identificação pela retirada das genitálias $\left(\sigma^{*}\right)$ e identificação sob microscópio estereoscópico. A técnica utilizada consiste em fazer uma pequena incisão nos pleuritos do pigidio para retirada do edeago, após o que, este é anexado a seco ao exemplar em uma outra etiqueta. O material coletado está depositado na Coleção Entomógica do INPA.

\section{RESULTADOS}

Entre julho de 1990 e junho de 1991 foram coletados 251 indivíduos distribuídos em 9 gêneros e 18 espécies: Cyclocephala colasi Endrödi, 1964 (128 indivíduos); Cyclocephala bicolor Castelnau, 1840 (26); Cyclocephala affinis Endrödi, 1966 (14); Cyclocephala hardyi Endrödi, 1975 (10); Cyclocephala testacea Burmeister, 1847 (7); Cyclocephala mecynotarsis Hohne, 1923 (1); Cyclocephala ocellata Burmeister, 1847 (1); Cyclocephala variabilis Burmeister, 1847 (1); 
Cyclocephala verticalis Burmeister, 1847 (1); Stenocrates carbunculus Prell, 1938 (2); Stenocrates ligneus Arrow, 1911 (2); Dyscinetus dubius Olivier, 1789 (7); Megaceras stuebeli Kirsch, 1885 (7); Strategus aloeus Linnaeus, 1758 (3); Coelosis biloba Linnaeus, 1767 (1); Megasoma actaeon Linnaeus, 1758 (2); Ligyrus ebenus De Geer, 1774 (36) e Oxyligyrus zoilus Olivier, 1789 (2) (Tab. 1).

Nota-se pela distribuição das espécies que algumas aparecem em determinadas épocas do ano, o que deve estar relacionado às condições ambientais daquele período, tais como os períodos chuvoso (dezembro a maio) e seco (junho a novembro).

A partir das identificações das espécies, determinou-se a abundância de machos e fềmeas dos Dynastinae. $\mathrm{Na}$ distribuição ao longo do ano, observa- se uma predominância de fềmeas.

Entre os Dynastinae ocorridos na Fazenda Aruanã, Cyclocephala correspondeu a 189 indivíduos de 9 espécies diferentes, sendo $75 \%$ do total desta subfamília. Das 9 espécies coletadas, 3 tiveram maior número de indivíduos. Entre estas, Cyclocephala colasi apresentou registros em quase todos os meses, sendo que as maiores distribuições corresponderam aos meses de fev-mar, um pico observado para a espécie e para o gênero.

Para os Dynastinae coletados, Ligyrus apresentou 36 indivíduos de uma única espécie, ou seja, $14,3 \%$ do total desta subfamília.

\section{DISCUSSÃO}

Os dados apresentados de julho/ 90 a junho/91 ilustram uma parte do que pode ser a comunidade de

Tabela 1. Dinastíneos coletados na Fazenda Aruanã, Itacoatiara, AM, no período de junho/1990 a julho/1991.

\begin{tabular}{|c|c|c|c|c|c|c|c|c|c|c|c|c|c|c|c|c|}
\hline TRIBO & ESPÉCIE & $\mathrm{J}$ & A & $\mathbf{s}$ & 0 & $\mathbf{N}$ & D & 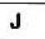 & $\mathbf{F}$ & M & A & M & $\mathrm{J}$ & TT & $\sigma^{*}$ & $q$ \\
\hline \multirow{12}{*}{ Cyclocephalini } & Cyclocephala colasl & 3 & 1 & 4 & 8 & 4 & 6 & 4 & 36 & 56 & 5 & 1 & & 128 & 9 & 119 \\
\hline & Cyclocephala bicolor & 9 & 1 & 12 & 1 & & & & & & 1 & & 2 & 26 & 1 & 25 \\
\hline & Cyclocephala affinis & & & & & & 1 & 1 & & & & 3 & 9 & 14 & 1 & 13 \\
\hline & Cyclocephala hardyi & & 2 & & & & & & 2 & & 1 & & 5 & 10 & 3 & 7 \\
\hline & Cyclocephata testacea & 3 & 2 & 1 & & & & & & & & & 1 & 7 & 0 & 7 \\
\hline & Cyclocephala mecynotarsis & & & 1 & & & & & & & & & & 1 & 1 & 0 \\
\hline & Cyclocephala oceiiata & & & 1 & & & & & & & & & & 1 & 0 & 1 \\
\hline & Cyclocephala variabilis & & & & & & 1 & & & & & & & 1 & 0 & 1 \\
\hline & Cyclocephala verticalis & & & 1 & & & & & & & & & & 1 & 0 & 1 \\
\hline & Stenocrates carbunculus & & & 1 & & & 1 & & & & & & . & 2 & 1 & 1 \\
\hline & Stenocrates ligneus & & & & 1 & 1 & & & & & & & & 2 & 0 & 2 \\
\hline & Dyscinetus dubius & & & & & & 5 & & 1 & 1 & & & & 7 . & 2 & 5 \\
\hline \multirow[t]{3}{*}{ Oryctini } & Megaceras stuebell & & & 3 & & & & & & 2 & & & 2 & 7 & 3 & 4 \\
\hline & Strategus aloeus & 1 & 2 & & & & & & & & & & & 3 & 0 & 3 \\
\hline & Coeiosis biloba & & & & & & & & & & & & 1 & 1 & 1 & 0 \\
\hline Dynastini & Megasoma actaeon & & & 2 & & & & & & & & & & 2 & 2 & 0 \\
\hline \multirow[t]{2}{*}{ Pentodontini } & Ligyrus ebenus & 4 & 10 & & & & 12 & 9 & 1 & & & & & 36 & 19 & 17 \\
\hline & Oxylligyrus zoilus & & & & & 2 & & & & & & & & 2 & 1 & 1 \\
\hline Total & & 20 & 18 & 26 & 10 & 7 & 26 & 14 & 40 & 59 & 7 & 4 & 20 & 251 & 44 & 207 \\
\hline
\end{tabular}


dinastíneos de uma área de floresta de terra firme. A abundância de certas espécies de Cyclocephala para esse período, assim como a pequena ocorrência de outras espécies pode ser uma resposta à forma utilizada de captura e/ou também do local escolhido. $\mathrm{O}$ mesmo pode-se dizer a respeito da predominância de fêmeas em relação aos machos (Tab. 1) mostrando que a luz exerce maior efeito atrativo nas fềmeas. O gênero Cyclocephala é ainda pouco conhecido, sendo de larga distribuição geográfica na Amazônia porém com hábitos desconhecidos para a maioria das espécies. Esta aparente dominância de algumas espécies $(C$. colasi e $C$. bicolor) parece ser referente a capacidade destas espécies em tolerar modificações ambientais adaptando-se às condições impostas e colonizando novos nichos. Vale dizer que estas espécies são freqüentemente coletadas em localidades próximas à periferia de Manaus, mesmo onde há iluminação deficiente ou fraca e ainda porções de mata por perto. E ainda tem-se o ciclo das chuvas que rege toda a vida na floresta mostrando em duas estações, condições ambientais que podem ser favoráveis a algumas espécies e desfavoráveis a outras.

A Fazenda Aruanã sendo uma área perturbada, visto que já sofreu um certo impacto quando da implantação de pasto, permite traçar alguns dados úteis sobre a fauna de dinastíneos da região. Sendo portanto uma área que já foi alterada, apesar de o ser em pequena escala em relação aos limites extensos da fazenda, pode ter favorecido determinadas espécies que encontraram novas condições antes não existentes. Como não temos a situação inicial e não há trabalhos com esse grupo na região, os resultados permitem pouca comparação nesse sentido. Alguns dos gêneros capturados em maior quantidade são considerados pragas agrícolas em outros locais, como é o caso de Cyclocephala, Ligyrus e Strategus. Este reconhecimento pode vir a ser importante no caso da instalação de novos plantios para a fazenda e outras regiões da Amazônia justamente pela facilidade em adaptar-se a condições modificadas que outras espécies não conseguem. Um exemplo deste fato está em que recentemente houve uma explosão populacional de Lygirus similis em um município próximo a Manaus ocasionando grandes danos em bananeiras (Garcia et al., 1994). Esta espécie ainda não tinha sido registrada como praga em plantas cultivadas.

A luz de mercúrio mostrou ter uma grande eficiência para as coletas de Dynastinae, visto que este método já era utilizado para lepidópteros e coleópteros passalídeos. Porém, apenas não se havia contabilizado o quanto este tipo de coleta pode trazer resultados a nível genérico $\mathrm{e}$ específico para os escarabeídeos. Como estes têm seu desenvolvimento em troncos podres fica difícil encontrar adultos já na fase de abandono deste local. Para isso a luz aparece como um fator atrativo até agora importantíssimo, visto que não se conhece este grupo, e ainda exemplares têm sido coletados freqüentemente em outros tipos de luz, 
até de fraca intensidade.

Os conhecimentos sobre a fauna de escarabeídeos nas regiões de florestas tropicais das Américas ainda são poucos. As últimas citações e descrições sobre espécies novas das várias coletas com armadilhas luminosas e iscas são principalmente do México e Panamá, principalmente o primeiro. Palacios-Rios et al. (1990) em seu levantamento encontraram alguns gêneros em comum aos coletados na Fazenda Aruanã porém de espécies diferentes. Entretanto, utilizaram armadilha luminosa das 19 às 23 horas além de outros métodos de captura.

Morón (1979) relacionou 6 gêneros da tribo Cyclocephalini que ocorrem no México, 3 deles registrados na Fazenda Aruanã: Cyclocephala, Stenocrates e Dyscinetus, sendo o primeiro deles com uma representação mais ampla e diferenciação por altitude. Apesar de utilizarem um período mais curto de coleta, também encontraram várias espécies para os locais amostrados.

Em Dynastinae muitas espécies estão sendo descritas e há outras para serem investigadas sendo incipientes alguns dados sobre estes coleópteros na região amazônica. A possibilidade de levantamentos de fauna permite conhecimentos amplos para o grupo principalmente na distribuição geográfica, fornecendo dados quantitativos $\mathrm{e}$ qualitativos sobre as espécies encontradas. Em relação aos Dynastinae, por exemplo, Ratcliffe (1978) sugere que Stenocrates seria um gênero evolutivamente recente passando por um processo de especiação e expansão "ativas" a partir das sucessivas descrições de espécies novas nos últimos anos. Este processo pode se repetir para outros gêneros, principalmente Cyclocephala, onde têm sido grande o número de espécies novas descritas nos últimos anos. Endrödi (1985) fez uma revisão mundial para Dynastinae e descreveu mais espécies para esse gênero. Deve-se ressaltar que as diferentes espécies da Amazônia brasileira e de outros países sulamericanos ainda são pouco conhecidas e que somente a partir de levantamentos e coletas regulares poder-se-á ter um conhecimento melhor das espécies de Scarabaeidae presentes nestes locais.

\section{AGRADECIMENTOS}

Os autores agradecem aos Engenheiros Agrônomos Sérgio Vergueiro e Gabriel Teixeira de Paula Neto (Diretor da Fazenda Aruanã) pelo apoio dado durante a execução deste trabalho e à pesquisadora Catarina da Silva Motta (CPEN / INPA) pela oportunidade para realizá-lo.

\section{Bibliografia Citada}

Aguiar, N. O.; Bührnheim, P.F. 1992. Pseudoscorpiones (Arachnida) em associação forética com Passalidae (Insecta, Coleoptera) no Amazonas, Brasil. Amazoniana 12 (2): 187-205.

Blackwelder, R.E. 1944. Checklist of the Coleopterous Insects of Mexico, Central America, The West Indies and South America. Part 2 . Bulletin of the United States National Museum 185, Scarab., 189-265.

Bührnheim, P.F.; Aguiar, N.O. 1991. Passalídeos (Coleoptera) da Ilha de Maracá, Roraima. Acta Amazonica 21: 25-33.

Dantas, M. 1979. Pastagens da Amazônia Central: Ecologia e fauna do solo. Acta 
Amazonica 9(2): Suplemento, 54 p.

Endrödi, S. 1985. The Dynastinae of the world. Dr. W. Junk Publishers, Budapest, Hungary, 800p. 56 estampas.

Garcia, M.V.B.; Ronchi-Teles, B.; Pamplona, A.M.S.R.; Andreazze, R. 1994. Ocorrência de Ligyrus similis (Coleoptera, Scarabaeidae) como Praga da Bananeira no Estado do Amazonas. Resumos: XIII Congresso Brasileiro de Fruticultura 173, Salvador, BA.

Gallo, D.; Nakano, O.; Silveira-Neto, S.; Carvalho, R.P.L.; Batista, G.C. DE; Berti-Filho, E.; Parra, J.R.P.; Zucchi, R.A.; Alves, S.B. 1978. Manual de Entomologia Agricola. Ed. Agronômica Ceres Ltda. São Paulo. 531 p.

Janssens, A. 1946. Contribution a l'étude des Coléoptères Lamellicornes Coprophages. Bulletin du Musée royal d'Histoire naturelle de Belgique. 22(12):1-13.

.... 1949. Contribution a l'étude des Coléoptères Lamellicornes. Bulletin Institut royal des Sciences naturelles de Belgique. 25(15):1-30.

Laroca, S.; Mielke, O.H.H. 1975. Ensaios sobre ecologia de comunidade em Sphingidae na Serra do Mar, Paraná, Brasil (Lepidoptera). Revista Brasileira de Biologia. 35(1): 1-19.

Lima, A.M. DA C. 1953. Insetos do Brasil. 8 (Coleópteros) 2a parte. 323 p. 103 figs. Escola Nacional de Agronomia. Rio de Janeiro.

Morón, R.M.A. 1979. La tribu Cyclocephalini (Coleoptera, Melolonthidae, Dynastinae) en México. Folia Entomologica Mexicana 42: 72-73.
.... 1981. Fauna de coleópteros Melolonthidae de la Reserva de la Biosfera "La Michilia", Durango, México. Folia Entomologica Mexicana 50: 3-69.

.... 1984. Escarabajos-200 miliones de años de evolucion. Instituto de Ecologia. Mexico. D.F. 132p.

.... 1985. Los insectos degradadores, un factor poco estudiado en los bosques de Mexico. Folia Entomologica Mexicana 65: 131-137.

Morón, R.M.A.; Villalobos,F.J.; Deloya,C. 1985. Fauna de coleópteros lamelicornios de Boca del Chajul, Chiapas, Mexico. Folia Entomologica Mexicana 66: 57-118.

Palacios-Rios, M.; Rico-Gray, V.; FuentesUENTES, E. 1990. Inventario preliminar de los Coleoptera Lamellicornia de la zona de Yaxchilan, Chiapas, Mexico. Folia Entomologica Mexicana No.78: 49-60.

Ratcliffe, B.C. 1978. New species of Stenocrates from Brazil (Coleoptera: Scarabaeidae). Manaus, Acta Amazonica 8(3): 489-495.

Ritcher, P.O. 1958. Biology of Scarabaeidae. Annual Review of Entomology, 3: 311-334.

Williams, C.B. 1936. The influence of moonlight on the activity of certain nocturnal insects, particularly of the family Noctuidae, as indicated by a light trap. Phillophs Transactions of the Royal Society of London (B) 226: 357-389.

Williams, C.B.; Singh, B.P. 1951. Effect of moonlight on insect activity. Nature, 187 , no. 4256, p. 853 . 